\title{
CAR-T cell therapy in less than 2 year old children with infant acute lymphoblastic leukemia : case reports and review of the literature
}

Laurent Cimasoni ${ }^{1}$, Julie Sartoretti ${ }^{1}$, Francesco Ceppi ${ }^{2}$, Shannon Maude ${ }^{3}$, Nicole Bodmer ${ }^{4}$, Mathias Hauri-Hohl ${ }^{4}$, Peter Bader ${ }^{5}$, Frederic Baleydier ${ }^{6}$, Fanette Bernard ${ }^{1}$, and Marc Ansari $^{7}$

${ }^{1}$ Hôpitaux Universitaires de Genève

${ }^{2} \mathrm{CHUV}$

${ }^{3}$ The Children's Hospital of Philadelphia

${ }^{4}$ Universitats-Kinderspital Zurich

${ }^{5}$ Klinikum der Johann Wolfgang Goethe-Universitat Frankfurt

${ }^{6}$ Hopitaux Universitaires de Geneve Hopital des enfants

${ }^{7}$ Hôpital universitaire de Genève

July 13, 2020

\begin{abstract}
Infant acute lymphoblastic leukemia (infant ALL) is known for its poor prognosis. Current treatment protocols include aggressive chemotherapy and allogeneic stem cell transplantation but prognosis remains poor. Immunotherapy is a new approach with promising results. CAR-T cell therapy has recently been approved for relapsed or refractory childhood ALL but lacks documentation regarding infant ALL. We describe here two cases of patients suffering from infant ALL who were treated with CAR-T cell therapy, the feasibility and efficiency of treatment and their outcome.
\end{abstract}

Title:

CAR-T cell therapy in less than 2 year old children with infant acute lymphoblastic leukemia : case reports and review of the literature

Authors:

L. Cimasoni ${ }^{1}$, J. Sartoretti ${ }^{1}$, F. Ceppi ${ }^{2}$, SL. Maude ${ }^{3}$, N. Bodmer ${ }^{4}$, M. Hauri-Hohl ${ }^{4}$, P. Bader ${ }^{5}$, F. Baleydier ${ }^{1}$, F. Bernard ${ }^{1}$, M. Ansari ${ }^{1}$

${ }^{1}$ Pediatric Oncology and Hematology Unit, Department of Pediatrics, Geneva Children's Hospital, University Hospital of Geneva, Geneva, Switzerland; and Cansearch Research Laboratory, University of Geneva.

${ }^{2}$ Pediatric Hematology-Oncology Unit, Division of Pediatrics, Department Woman-Mother-Child, Lausanne University Hospital, Lausanne, Switzerland.

${ }^{3}$ Division of Oncology, The Children's Hospital of Philadelphia, Philadelphia, PA.

${ }^{4}$ Pediatric Stem Cell Transplantation Unit, Department of Pediatrics, Zurich Children's Hospital, Zurich, Switzerland 
${ }^{5}$ Division for Stem Cell Transplantation and Immunology, Department for Children and Adolescents, University Hospital, Goethe University, Frankfurt/Main, Germany

Acknowledgements: R. Renella² ${ }^{2}$ M. Beck-Popovic ${ }^{2}$, S. Kroiss ${ }^{4}$, JP Bourquin ${ }^{4}$

Corresponding author :

Dr Laurent Cimasoni

Hôpitaux universitaires de Genève (HUG)

Département d'onco-hématologie pédiatrique

Rue Willy-Donzé 6

1205 Genève / CH

laurent.cimasoni@hcuge.ch/ +4179.885.90.85

Word Count : Abstract 80 words, Main text 1494 words

Table and Figure : Table 1, Figure 1 (1A and 1B)

Key words : Infant ALL, CAR-T cell therapy, Tisagenlecleucel, case study

Abbreviations :

\begin{tabular}{ll}
\hline Infant ALL & infant acute lymphoblastic leukemia \\
\hline HSCT & hematopoietic stem cell transplantation \\
MRD & Minimal residual disease \\
HUG & Geneva University Hospital \\
CAR & chimeric antigen receptor \\
\hline
\end{tabular}

Abstract:

Infant acute lymphoblastic leukemia (infant ALL) is known for its poor prognosis. Current treatment protocols include aggressive chemotherapy and stem cell transplantation but prognosis remains poor. Immunotherapy is a new approach with promising results. CAR-T cell therapy has recently been approved for relapsed or refractory childhood ALL but lacks documentation regarding infant ALL. We describe here two cases of patients suffering from infant ALL who were treated with CAR-T cell therapy, the feasibility and efficiency of treatment and their outcome.

Introduction

Infant acute lymphoblastic leukemia (ALL), defined as occurring before 12 months of age, is a rare disease, occurring in 40 cases per million births ${ }^{1}$. In up to $80 \%$ of cases, a KMT2A gene rearrangement, or KMT2A-r, occurs in utero and leads to a rapid progression to leukemia ${ }^{2}$. Very early relapse is common, leading to a 5 -year overall survival of about $50 \%$, and below $15 \%$ for high risk groups ${ }^{3,4}$.

There is hope that cancer immunotherapy may provide the long awaited breakthrough in treating these children. As such, CD19 directed chimeric antigen receptor (CAR)-T cells have recently been approved for relapsed or refractory childhood ALL, but their usage in infant ALL is not yet well documented.

We present two cases of infant ALL treated with this new therapy.

Case 1 (figure 1A)

This patient was diagnosed with high risk infant B-ALL at 5 months of age. 
Minimal residual disease (MRD) was negative after induction therapy according to the Interfant 06 protocol $^{5}$, but cerebrospinal fluid (CSF) became positive at the start of consolidation, and bone marrow MRD became detectable three weeks later.

He proceeded to a 10/10 HLA matched umbilical cord blood transplant after a busulfan-fludarabine and thiotepa conditioning regimen, according to the FORUM study ${ }^{6}$.

Unfortunately, he developed a bone marrow relapse two months after the transplant. It was decided to proceed to CAR-T cell therapy with the close collaboration of the hospital of Frankfurt, Germany, as Tisagenlecleucel was not available yet in Switzerland. At 13 months of age and $8.1 \mathrm{~kg}$, he was the smallest patient referred there for CAR-T cell therapy. Two apheresis were needed to collect enough cells, and in waiting for the re-infusion, he received a low intensity bridging chemotherapy according to the Frankfurt protocol $^{7}$.

He then went back to Frankfurt and received the CAR-T cells at a dose of $2 \times 10 \mathrm{e} 6 / \mathrm{kg}$. He did not show side effects of the T cells, but developed bacteremia due to an infection of the apheresis catheter. MRD was negative after CAR-T cell therapy.

Unfortunately, the CAR-T cells started decreasing and the patient presented a second bone marrow relapse as well as a subcutaneous chloroma four months after the infusion.

The Novartis facility had enough CAR-T cells to allow a new administration with a similar dose $(1.8 \mathrm{x} 10 \mathrm{e} 6 / \mathrm{kg})$. This took place in the Children's Hospital of Zurich, Switzerland, without any complications, and his MRD became negative again. The circulating CAR-T cell numbers however showed a progressive decline over the next months, and it was decided to proceed to a second HSCT in Geneva, after a Fludarabine, Thiotepa and Treosulfan conditioning regimen.

He is currently in remission with negative MRD, 9 months after his second transplant.

Case 2 (figure 1B)

The second patient was diagnosed with high risk infant B-ALL at less than two months of age. During consolidation according to the Interfant 06 protocol, her MRD became positive (10e-4) and she presented a very early CNS relapse.

Blinatumomab was started as a bridge to stem cell transplant, but her disease progressed with a bone marrow and CNS relapse, and she proceeded to a 10/10 matched cord blood stem cell transplant from an unrelated donor, after conditioning according to the FORUM protocol ${ }^{6}$.

Unfortunately, only a month after transplant, she presented a new medullary and CNS relapse. She was elected to receive CAR-T cell therapy, and was started on the Frankfurt ALL relapse protocol ${ }^{7}$. As soon as the absolute lymphocyte number reached 500/ul, she was transferred to the Children's Hospital of Zurich for the apheresis, at 12 months of age and with a weight of $9.5 \mathrm{~kg}$. The procedure had to be repeated twice to collect enough $\mathrm{T}$ cells.

In the meantime, the CNS infiltration progressed with meningeal chloromas, facial palsy and then impaired neurological status.

Tisagenlecleucel was infused at 2x10e6 cells/kg after a conditioning with Fludarabine and Cyclophosphamide. She developed a grade IV cytokine release syndrome which was treated with three doses of tocilizumab. She also presented a grade I CAR-T encephalopathy, which she seemed to recover from after a week. However her CNS disease progressed further, and she passed away less than a month after receiving the CAR-T cells.

Discussion

We report the first two cases of high risk infant B-ALL treated with Tisagenlecleucel (Table 1).

CAR-T cell therapy has shown promising results in pediatric ALL. It involves autologous $\mathrm{T}$ cells which are genetically modified to express a chimeric antigen receptor (CAR), usually targeting CD19. Tisagenlecleu- 
cel is a commercially available CAR-T cell developed by the University of Pennsylvania and by Novartis which has been approved by the FDA in 2017 and by the European and Swiss agencies in 2018 for refractory/relapsed B-ALL in young people aged 3 to 25 years. It is a second-generation CAR, meaning it includes

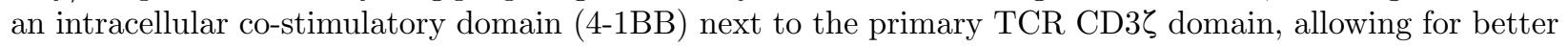
cellular expansion and survival.

The production starts with a leukapheresis to collect $\mathrm{T}$ cells. The prior treatments may lower white blood cell and lymphocyte counts, making it harder for the apheresis centrifuge to separate the cell populations ${ }^{8,9}$ and get a sufficient yield ${ }^{10}$. To alleviate this problem, some groups perform apheresis early in the treatment, with freezing of the cells.

In a Novartis facility, the cells are transduced with a lentiviral vector to express the CAR and are expanded, a process which takes just over three weeks. After a lymphodepleting chemotherapy, the cells are reinfused to the patient at a recommended dose of 0.2 to 5 x10e $6 \mathrm{~T}$ cells per $\mathrm{kg}$ in children ${ }^{11}$.

Early studies showed CR rates in relapsed pediatric and young adult B-ALL of 79 to $93 \%{ }^{12-15}$, and recent data on follow-up show remissions of over 60 months in non-CNS3 patients ${ }^{16}$. CAR-T cells also cross the blood-brain barrier and are able to target CSF disease ${ }^{17}$. The two main causes of treatment failure are early loss of CAR-T cells and CD19-negative relapses.

The differences in CAR-T construct, conditioning therapy and previous treatments make it difficult for clinicians to decide exactly when and how it should be used. For instance, the role of HSCT after CAR-T therapy is still an open question ${ }^{10,18-19}$.

As for infant ALL, it is worth noting that the trials which led to the approval of Tisagenlecleucel in children only included patients over 1 year of age. Seattle Children's Hospital recently reported the largest cohort of relapsed or refractory infant ALL treated with CAR-T cells with 18 patients, aged 14 to 40 months at enrollment, 16 of which received the treatment, with an MRD negative CR rate of $93 \%$ and one year OS of $71 \%^{20}$. Of the 14 patients who achieved MRD-negative CR, 6 went on to subsequent HSCT.

In our situation, the CAR-T treatment had to be repeated for the first patient, but it induced an MRD negative response both times. Careful surveillance of the CAR-T cell numbers then enabled us to proceed to a timely second stem cell transplant, with the patient remaining in complete remission with negative MRD until now and in excellent condition.

As for the second child, the disease was never in molecular remission but instead progressed in spite of the treatment, especially the CNS involvement which ultimately caused her death. Although less than two months went by from the day of the apheresis to the infusion of the cells, this time constraint remains an issue. Also, this child initially received Blinatumomab in an effort to reach negative MRD for transplant, and recent data suggest that prior use of Blinatumomab might decrease the efficacy of CD19 targeted CAR-T cells ${ }^{21}$.

The low patient weight and previous chemotherapies rendered the apheresis challenging, but these cases show that the procedure can be safely performed.

However, practical challenges remain, with relatively few manufacturing facilities worldwide, requiring tight coordination with the pharmaceutical company. In situ CAR-T production, available in some University Hospitals, alleviates these hurdles.

The cost of this therapy is also an issue. Compared to standard treatment, CAR-T cell therapy in pediatric ALL leads to an estimated increased cost of $\$ 528^{\prime} 200$, or $\$ 64^{\prime} 600$ per quality-adjusted life-year ${ }^{22}$. This is usually considered cost-effective, but is not easily affordable.

Finally, relapses may still occur in up to $50 \%$ of patients, emphasizing the importance of the ongoing research into new generations of CAR-T constructs, with better persistence and/or dual targets to avoid CD19 negative relapse. 
Conclusion

We report the first two cases of infant ALL treated with Tisagenlecleucel.

These cases underline the practical challenges involved, with the apheresis notably still quite challenging in infants. However, we demonstrate its feasibility in two cases of a disease once thought to be incurable: infant ALL relapsed after HSCT. It is our hope that with the more widespread use of CAR-T cells, progresses in their production and more efficacious constructs, the process will become more streamlined and hence easier for patients and their families. This may even allow treatment earlier in the disease course, sparing toxicities and allowing for more efficient treatment.

Conflict of Interest Statement

SL Maude: clinical trial support: Novartis; consulting, advisory boards, or study steering committee: Novartis, Kite, Wugen

References

1. Brown P. Treatment of infant leukemias: challenge and promise.Hematol Educ Program Am Soc Hematol Am Soc Hematol Educ Program . 2013;2013:596-600. doi:10.1182/asheducation-2013.1.596

2. Brown P, Pieters R, Biondi A. How I treat infant leukemia.Blood . 2019;133(3):205-214. doi:10.1182/blood-2018-04-785980

3. Pieters R, Schrappe M, De Lorenzo P, et al. A treatment protocol for infants younger than 1 year with acute lymphoblastic leukaemia (Interfant-99): an observational study and a multicentre randomised trial. Lancet Lond Engl . 2007;370(9583):240-250. doi:10.1016/S0140-6736(07)61126-X

4. Hilden JM, Dinndorf PA, Meerbaum SO, et al. Analysis of prognostic factors of acute lymphoblastic leukemia in infants: report on CCG 1953 from the Children's Oncology Group. Blood . 2006;108(2):441-451. doi:10.1182/blood-2005-07-3011

5. INTERFANT-06, International collaborative treatment protocol for infants under one year with acute lymphoblastic or biphenotypic leukemia, Amendment 8.0: April 6, 2017

6. Peters C, et al, TBI or chemotherapy based conditioning for children and adolescents with ALL: a prospective randomized multicenter-study 'FORUM' on behalf of the AIEOP-BFM-ALL-SG, IBFM-SG, INTREALL-SG and EBMT-PD-WP, EHA Library Abstract S102. June 2020

7. Willasch AM, Salzmann-Manrique E, Bader P. et al, Treatment of relapse after allogeneic stem cell transplantation in children and adolescents with ALL: the Frankfurt experience. Bone Marrow Transplant. 2017 Feb;52(2):201-208

8. Fesnak A, Lin C, Siegel DL, Maus MV. CAR-T cell therapies from the transfusion medicine perspective. Transfus Med Rev . 2016;30(3):139-145. doi:10.1016/j.tmrv.2016.03.001

9. Ceppi F, Rivers J, Annesley C, et al. Lymphocyte apheresis for chimeric antigen receptor T-cell manufacturing in children and young adults with leukemia and neuroblastoma. Transfusion. 2018;58(6):1414-1420. doi:10.1111/trf.14569

10. Kansagra AJ, Frey NV, Bar M, et al. Clinical utilization of Chimeric Antigen Receptor T-cells (CART) in B-cell acute lymphoblastic leukemia (ALL)-an expert opinion from the European Society for Blood and Marrow Transplantation (EBMT) and the American Society for Blood and Marrow Transplantation (ASBMT). Bone Marrow Transplant . 2019;54(11):1868-1880. doi:10.1038/s41409-019-0451-2

11. KYMRIAH@ [prescribing information]. East Hanover, NJ: Novartis Pharmaceuticals Corp; 2018.

12. Maude SL, Frey N, Shaw PA, et al. Chimeric antigen receptor T cells for sustained remissions in leukemia. N Engl J Med . 2014;371(16):1507-1517. doi:10.1056/NEJMoa1407222 
13. Gardner RA, Finney O, Annesley C, et al. Intent-to-treat leukemia remission by CD19 CAR T cells of defined formulation and dose in children and young adults. Blood . 2017;129(25):3322-3331. doi:10.1182/blood2017-02-769208

14. Maude SL, Laetsch TW, Buechner J, et al. Tisagenlecleucel in Children and Young Adults with B-Cell Lymphoblastic Leukemia. N Engl J Med . 2018;378(5):439-448. doi:10.1056/NEJMoa1709866

15. Ceppi F, Renella R, Diezi M, et al. [Recent advances and future directions in CAR-T cell therapy in pediatric oncology]. Rev Med Suisse . 2019;15(Nº 632-633):85-91.

16. Sa G, Sl M, Tw L, Ma P. Updated Analysis of the Efficacy and Safety of Tisagenlecleucel in Pediatric and Young Adult Patients with Relapsed/Refractory Acute Lymphoblastic Leukemia: The ELIANA Trial. $: 15$.

17. Grupp SA, Kalos M, Barrett D, et al. Chimeric Antigen Receptor-Modified T Cells for Acute Lymphoid Leukemia. N Engl J Med . 2013;368(16):1509-1518. doi:10.1056/NEJMoa1215134

18. DiNofia AM, Maude SL. Chimeric Antigen Receptor T-Cell Therapy Clinical Results in Pediatric and Young Adult B-ALL. HemaSphere . 2019;3(4):e279. doi:10.1097/HS9.0000000000000279

19. Summers C, Annesley C, Bleakley M, et al. Long Term Follow-up after SCRI-CAR19v1 Reveals Late Recurrences As Well As a Survival Advantage to Consolidation with HCT after CAR T Cell Induced Remission. Presented at: ASH Annual Meeting and Exposition; Dec. 1-4, 2018; San Diego. Abstract 967.

20. Annesley C, Summers C, Pulsipher MA, et al. Clinical Experience of CAR T Cell Immunotherapy for Relapsed and Refractory Infant ALL Demonstrates Feasibility and Favorable Responses. Blood . 2019;134(Supplement_1):3869-3869. doi:10.1182/blood-2019-131447

21. Pillai V, Muralidharan K, Meng W, et al. CAR T-cell therapy is effective for CD19-dim Blymphoblastic leukemia but is impacted by prior blinatumomab therapy. Blood Adv . 2019;3(22):3539-3549. doi:10.1182/bloodadvances.2019000692

22. Sarkar RR, Gloude NJ, Schiff D, Murphy JD. Cost-Effectiveness of Chimeric Antigen Receptor T-Cell Therapy in Pediatric Relapsed/Refractory B-Cell Acute Lymphoblastic Leukemia. JNCI J Natl Cancer Inst . 2018;111(7):719-726. doi:10.1093/jnci/djy193

\section{Hosted file}

Table 1.docx available at https://authorea.com/users/341531/articles/468409-car-t-celltherapy-in-less-than-2-year-old-children-with-infant-acute-lymphoblastic-leukemia-casereports-and-review-of-the-literature 
A

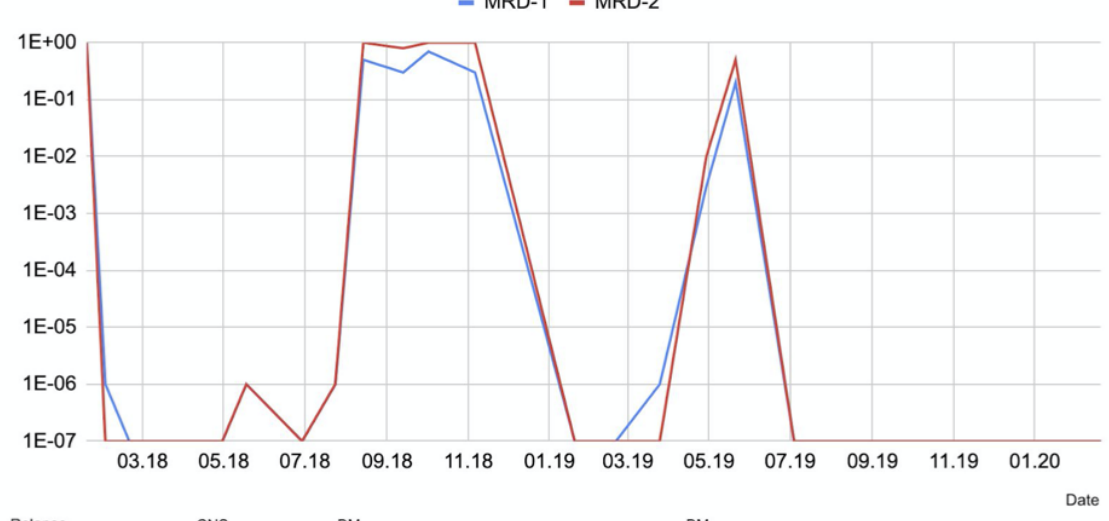

Relapse

CNS

$\stackrel{\uparrow}{\stackrel{\uparrow}{4} \text { CAR-T }}$

BM

$\frac{t}{C A R \cdot T} \stackrel{t}{\square S C T}$

B

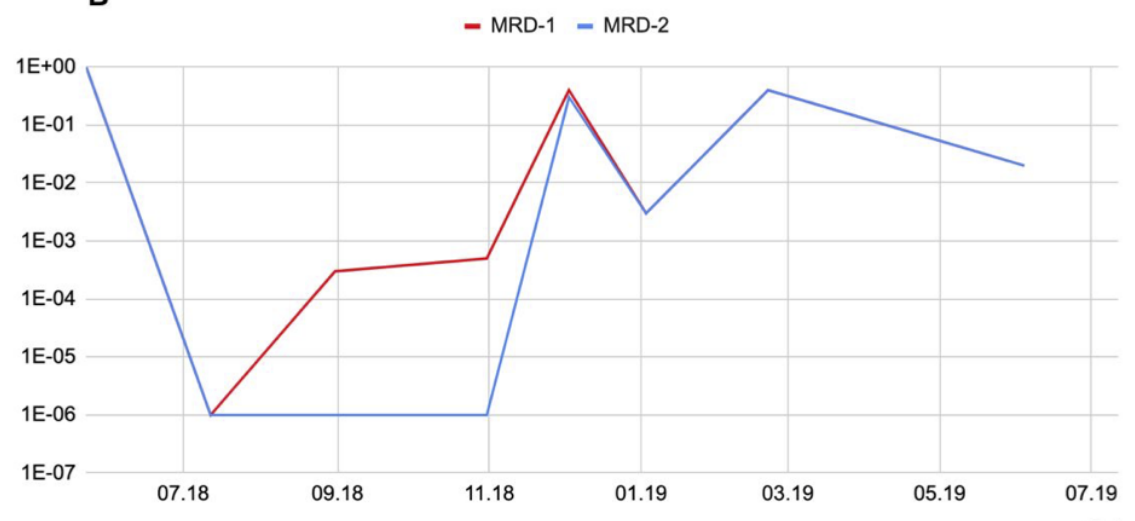

Relapse

CNS

$\mathrm{CNS}+\mathrm{BM}$

$\mathrm{CNS}+\mathrm{BM}$

Therapy

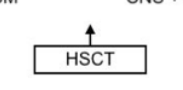

